\title{
The temperature-jump problem for a variable collision frequency model
}

L. B. Barichello, A. C. R. Bartz, M. Camargo, and C. E. Siewert

Citation: Physics of Fluids 14, 382 (2002); doi: 10.1063/1.1416192

View online: https://doi.org/10.1063/1.1416192

View Table of Contents: http://aip.scitation.org/toc/phf/14/1

Published by the American Institute of Physics

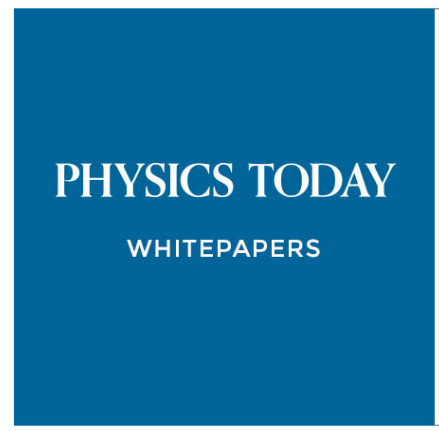

ADVANCES IN PRECISION

MOTION CONTROL

Piezo Flexure Mechanisms and Air Bearings

\section{READ NOW}

PRESENTED BY

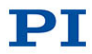




\section{The temperature-jump problem for a variable collision frequency model}

L. B. Barichello

Instituto de Matemática, Universidade Federal do Rio Grande do Sul, 91509-900 Porto Alegre, RS, Brazil

A. C. R. Bartz

Programa de Pós-Graduação em Matemática Aplicada, Universidade Federal do Rio Grande do Sul, 91509-900 Porto Alegre, RS, Brazil

M. Camargo ${ }^{\text {a) }}$

Programa de Pós-Graduação em Engenharia Mecânica, Universidade Federal do Rio Grande do Sul, 90050-170 Porto Alegre, RS, Brazil

C. E. Siewert

Mathematics Department, North Carolina State University, Raleigh, North Carolina 27695-8205

(Received 3 April 2001; accepted 14 August 2001)

An analytical version of the discrete-ordinates method is used here in the field of rarefied-gas dynamics to solve a version of the temperature-jump problem that is based on a linearized, variable collision frequency model of the Boltzmann equation. In addition to a complete development of the discrete-ordinates method for the application considered, the computational algorithm is implemented to yield accurate numerical results for three specific cases: the classical BGK model, the Williams model (the collision frequency is proportional to the magnitude of the velocity), and the rigid-sphere model. (C) 2002 American Institute of Physics. [DOI: 10.1063/1.1416192]

\section{INTRODUCTION}

The state of a gas can be described mathematically by a distribution function that satisfies the nonlinear Boltzmann equation. ${ }^{1-3}$ While, for example, Monte Carlo methods and computationally intensive iterative methods are ways of attempting to extract some physical information from the nonlinear Boltzmann equation, another approach that can be used when the density of particles is small (rarefied-gas dynamics) is to approximate the nonlinear Boltzmann equation by a so-called model equation. ${ }^{4}$ In recent years, we have seen an increased interest in the general area of rarefied-gas dynamics essentially because of applications to small-scale problems (for example, as related to micro-machines and high-speed disk drives) where the Boltzmann equation or a model equation is required in order to describe well gas-flow and heat-flow mechanisms. In this work, we take advantage of some recent mathematical and numerical improvements in the discrete-ordinates method in order to establish a series of high-quality results for the temperature-jump problem as based on a generalization of the standard BGK model.

Although the so-called BGK model ${ }^{5}$ introduced by Bhatnagar, Gross, and Krook has been the focus of the vast majority of mathematical studies in the general area of rarefiedgas dynamics, there exist numerous models that have been used to try to improve on the simplest form of the BGK model. One such approach ${ }^{6-11}$ is based on the variable collision frequency model (sometimes referred to as the generalized BGK model) since it has been shown ${ }^{9}$ better able to support some experimental observations. In a recent work ${ }^{12}$

${ }^{a)}$ Permanent address: Universidade Regional Integrada do Alto Uruguai e das Missões. the variable collision frequency model was used to solve the classical Kramers' problem, ${ }^{3}$ and so in this work we extend our use ${ }^{13}$ of the discrete-ordinates method ${ }^{14}$ to solve the temperature-jump problem ${ }^{15}$ for a general version of a linearized, variable collision frequency model of the Boltzmann equation. Here we base our notation on Williams' book; ${ }^{3}$ however, the papers of Cercignani ${ }^{7}$ and Loyalka and Ferziger $^{8}$ are the ones we consider to be the defining works on this subject of the variable collision frequency model. It therefore seems reasonable to refer to the general model equation used in this work as the CLF equation and to consider the BGK model (constant collision frequency), the Williams model (the collision frequency is proportional to the particle speed), and the rigid-sphere model as special cases that correspond to certain choices of the collision frequency.

To introduce the mathematical statement of the problem to be solved, we follow Williams ${ }^{3}$ and consider the defining balance equation to be

$$
\begin{aligned}
c \mu & \frac{\partial}{\partial x} h(x, \mathbf{c})+V(c) h(x, \mathbf{c}) \\
\quad & \int_{0}^{\infty} \int_{-1}^{1} \int_{0}^{2 \pi} c^{\prime 2} e^{-c^{\prime 2}} K\left(\mathbf{c}: \mathbf{c}^{\prime}\right) h\left(x, \mathbf{c}^{\prime}\right) d \chi^{\prime} d \mu^{\prime} d c^{\prime} .
\end{aligned}
$$

Here

$$
h(x, \mathbf{c}) \Rightarrow h(x, c, \mu, \chi)
$$

and 


$$
\begin{aligned}
K\left(\mathbf{c}: \mathbf{c}^{\prime}\right)= & \frac{1}{4 \pi} V(c) V\left(c^{\prime}\right)\left[\gamma_{0}+\gamma_{1} \mathbf{c} \cdot \mathbf{c}^{\prime}+\gamma_{2}\left(c^{2}-\omega\right)\right. \\
& \left.\times\left(c^{\prime 2}-\omega\right)\right],
\end{aligned}
$$

where

$$
\begin{aligned}
& \gamma_{0}=\frac{1}{V_{0}}, \\
& \gamma_{1}=\frac{3}{V_{2}}, \\
& \gamma_{2}=\frac{V_{0}}{V_{0} V_{4}-V_{2}^{2}},
\end{aligned}
$$

and

$$
\omega=\frac{V_{2}}{V_{0}}
$$

with

$$
V_{n}=\int_{0}^{\infty} V(c) c^{n+2} e^{-c^{2}} d c .
$$

In addition, $c$ is used, with dimensionless units, to denote the magnitude of the particle velocity vector $\mathbf{c}, x \geqslant 0$ is the spatial variable that measures (in dimensionless units) the distance from the wall, $V(c)$ is the collision frequency, and $\mu$ and $\chi$ are the two angular variables that define the direction (relative to the positive $x$ axis) of the velocity. In addition to Eq. (1) we consider the boundary condition at the wall written as

$$
h(0, c, \mu, \chi)-(1-\alpha) h(0, c,-\mu, \chi+\pi)-(\mathcal{I} h)(0)=0
$$

for $\mu \in(0,1], c \in[0, \infty)$, and $\chi \in[0, \pi]$ and

$$
h(0, c, \mu, \chi)-(1-\alpha) h(0, c,-\mu, \chi-\pi)-(\mathcal{I} h)(0)=0
$$

for $\mu \in(0,1], c \in[0, \infty)$, and $\chi \in[\pi, 2 \pi]$. Here

$$
\begin{aligned}
(\mathcal{I} h)(0)= & \frac{2 \alpha}{\pi} \int_{0}^{\infty} \int_{0}^{1} \int_{0}^{2 \pi} c^{\prime 3} e^{-c^{\prime 2}} h\left(0, c^{\prime},-\mu^{\prime}, \chi^{\prime}\right) \\
& \times \mu^{\prime} d \chi^{\prime} d \mu^{\prime} d c^{\prime}
\end{aligned}
$$

and $\alpha \in(0,1]$ is the accommodation coefficient. Our basic unknown $h(x, \mathbf{c})$ is the perturbation from an initial Maxwellian distribution that, due to the presence of the wall, is a component of the particle distribution function. In regard to Eqs. (6), we note that some fraction $1-\alpha$ of the particles is reflected specularly and that the remaining fraction $\alpha$ is reflected diffusely. In other words, the wall acts somewhat like a mirror and at the same time appears to absorb some of the particles and then re-emit them isotropically. Because there is no loss or supply of particles due to the presence of the wall, the boundary condition can be thought of as conservative. In addition to the boundary condition given by Eqs. (6), we note that, as will be discussed later, we must also impose a condition on $h(x, \mathbf{c})$ as $x$ tends to infinity.

\section{QUANTITIES OF INTEREST}

While our problem is defined in terms of the basic unknown $h(x, \mathbf{c})$, we require only two elementary integrals of $h(x, \mathbf{c})$. To be clear, we note that here we seek the temperature and density perturbations ${ }^{8}$ defined by

$$
N(x)=\frac{1}{\pi^{3 / 2}} \int_{0}^{\infty} \int_{-1}^{1} \int_{0}^{2 \pi} c^{2} e^{-c^{2}} h(x, c, \mu, \chi) d \chi d \mu d c
$$

and

$$
\begin{aligned}
T(x)= & \frac{2}{3 \pi^{3 / 2}} \int_{0}^{\infty} \int_{-1}^{1} \int_{0}^{2 \pi} c^{2} e^{-c^{2}}\left(c^{2}-3 / 2\right) \\
& \times h(x, c, \mu, \chi) d \chi d \mu d c,
\end{aligned}
$$

or

$$
N(x)=\frac{2}{\pi^{1 / 2}} \int_{0}^{\infty} \int_{-1}^{1} c^{2} e^{-c^{2}} \phi(x, c, \mu) d \mu d c
$$

and

$$
T(x)=\frac{4}{3 \pi^{1 / 2}} \int_{0}^{\infty} \int_{-1}^{1} c^{2} e^{-c^{2}}\left(c^{2}-3 / 2\right) \phi(x, c, \mu) d \mu d c,
$$

where

$$
\phi(x, c, \mu)=\frac{1}{2 \pi} \int_{0}^{2 \pi} h(x, c, \mu, \chi) d \chi
$$

is an azimuthal average. We can integrate Eqs. (1) and (6) over $\chi$ to find

$c \mu \frac{\partial}{\partial x} \phi(x, c, \mu)+V(c) \phi(x, c, \mu)$

$$
=\int_{0}^{\infty} \int_{-1}^{1} c^{\prime 2} e^{-c^{\prime 2}} K\left(c, \mu: c^{\prime}, \mu^{\prime}\right) \phi\left(x, c^{\prime}, \mu^{\prime}\right) d \mu^{\prime} d c^{\prime},
$$

for $x>0, \mu \in[-1,1]$ and $c \in[0, \infty)$, and

$$
\begin{aligned}
& \phi(0, c, \mu)-(1-\alpha) \phi(0, c,-\mu) \\
& -4 \alpha \int_{0}^{\infty} \int_{0}^{1} c^{\prime 3} e^{-c^{\prime 2}} \phi\left(0, c^{\prime},-\mu^{\prime}\right) \mu^{\prime} d \mu^{\prime} d c^{\prime}=0,
\end{aligned}
$$

for $\mu \in(0,1]$ and $c \in[0, \infty)$. Here

$$
\begin{aligned}
K\left(c, \mu: c^{\prime}, \mu^{\prime}\right)= & \frac{1}{2} V(c) V\left(c^{\prime}\right)\left[\gamma_{0}+\gamma_{1} c \mu c^{\prime} \mu^{\prime}\right. \\
& \left.+\gamma_{2}\left(c^{2}-\omega\right)\left(c^{\prime 2}-\omega\right)\right] .
\end{aligned}
$$

As Eqs. (1) and (6) are homogeneous, we must specify a driving term for the temperature-jump problem. We do this implicitly by requiring that $h(x, c, \mu, \xi)$ diverge as $x$ tends to infinity. More specifically, we impose the condition that the temperature perturbation satisfies the Welander condition ${ }^{16}$

$$
\lim _{x \rightarrow \infty} \frac{d}{d x} T(x)=K,
$$

where $K$ is considered specified. Now let

$$
V(c)=\sigma \eta(c),
$$


where $\sigma$ is a scale factor to be defined later and where $\eta(c)$ is a "shape factor" used to define the variable collision frequency. We also introduce

$$
\tau=\sigma x
$$

and

$$
Y(\tau, c, \mu)=\phi(\tau / \sigma, c, \mu)
$$

and rewrite our problem as

$$
\begin{aligned}
& c \mu \frac{\partial}{\partial \tau} Y(\tau, c, \mu)+\eta(c) Y(\tau, c, \mu) \\
& \quad=\int_{0}^{\infty} \int_{-1}^{1} c^{\prime 2} e^{-c^{\prime 2}} F\left(c, \mu: c^{\prime}, \mu^{\prime}\right) Y\left(\tau, c^{\prime}, \mu^{\prime}\right) d \mu^{\prime} d c^{\prime}
\end{aligned}
$$

for $\tau>0, \mu \in[-1,1]$ and $c \in[0, \infty)$, and

$$
\begin{aligned}
& Y(0, c, \mu)-(1-\alpha) Y(0, c,-\mu) \\
& \quad-4 \alpha \int_{0}^{\infty} \int_{0}^{1} c^{\prime 3} e^{-c^{\prime 2}} Y\left(0, c^{\prime},-\mu^{\prime}\right) \mu^{\prime} d \mu^{\prime} d c^{\prime}=0,
\end{aligned}
$$

for $\mu \in(0,1]$ and $c \in[0, \infty)$. Here

$$
\begin{aligned}
F\left(c, \mu: c^{\prime}, \mu^{\prime}\right)= & \frac{1}{2} \eta(c) \eta\left(c^{\prime}\right)\left[\beta_{0}+\beta_{1} c \mu c^{\prime} \mu^{\prime}\right. \\
& \left.+\beta_{2}\left(c^{2}-\omega\right)\left(c^{\prime 2}-\omega\right)\right],
\end{aligned}
$$

where

$$
\begin{aligned}
& \beta_{0}=\frac{1}{\eta_{2}}, \\
& \beta_{1}=\frac{3}{\eta_{4}}, \\
& \beta_{2}=\frac{\eta_{2}}{\eta_{2} \eta_{6}-\eta_{4}^{2}}
\end{aligned}
$$

and

$$
\omega=\frac{\eta_{4}}{\eta_{2}}
$$

with

$$
\eta_{n}=\int_{0}^{\infty} \eta(c) c^{n} e^{-c^{2}} d c .
$$

Now we let

$$
T_{*}(\tau)=T(\tau / \sigma) \text { and } N_{*}(\tau)=N(\tau / \sigma),
$$

and so we can write

$$
N_{*}(\tau)=\frac{2}{\pi^{1 / 2}} \int_{0}^{\infty} \int_{-1}^{1} c^{2} e^{-c^{2}} Y(\tau, c, \mu) d \mu d c
$$

and

$$
T_{*}(\tau)=\frac{4}{3 \pi^{1 / 2}} \int_{0}^{\infty} \int_{-1}^{1} c^{2} e^{-c^{2}}\left(c^{2}-3 / 2\right) Y(\tau, c, \mu) d \mu d c .
$$

Finally to complete the definition of our problem, we rewrite Eq. (16) as

$$
\lim _{\tau \rightarrow \infty} \frac{d}{d \tau} T_{*}(\tau)=\frac{K}{\sigma} .
$$

At this point we note that

$$
Z_{a}(\tau, c, \mu)=\left(c^{2}-5 / 2\right)[\tau-c \mu / \eta(c)]
$$

is a solution of Eq. (19) that is linear in $\tau$, and so we choose to decompose the required solution into a part that has the desired behavior as $\tau$ tends to infinity and a part that is bounded. We therefore write

$$
Y(\tau, c, \mu)=\frac{K}{\sigma}\left[Z(\tau, c, \mu)+Z_{a}(\tau, c, \mu)\right]
$$

and find from Eqs. (25) and (26), after using Eqs. (28) and (29), results for the density and temperature perturbations expressed in terms of the bounded component $Z(\tau, c, \mu)$, viz.

$$
\begin{aligned}
N_{*}(\tau)= & (K / \sigma)\left[-\tau+\frac{2}{\pi^{1 / 2}}\right. \\
& \left.\times \int_{0}^{\infty} \int_{-1}^{1} c^{2} e^{-c^{2}} Z(\tau, c, \mu) d \mu d c\right]
\end{aligned}
$$

and

$$
\begin{aligned}
T_{*}(\tau)= & (K / \sigma)\left[\tau+\frac{4}{3 \pi^{1 / 2}} \int_{0}^{\infty} \int_{-1}^{1} c^{2} e^{-c^{2}}\left(c^{2}-3 / 2\right)\right. \\
& \times Z(\tau, c, \mu) d \mu d c] .
\end{aligned}
$$

It follows now that we seek a solution $Z(\tau, c, \mu)$ that is bounded as $\tau$ tends to infinity and that satisfies

$c \mu \frac{\partial}{\partial \tau} Z(\tau, c, \mu)+\eta(c) Z(\tau, c, \mu)$

$$
=\int_{0}^{\infty} \int_{-1}^{1} c^{\prime 2} e^{-c^{\prime 2}} F\left(c, \mu: c^{\prime}, \mu^{\prime}\right) Z\left(\tau, c^{\prime}, \mu^{\prime}\right) d \mu^{\prime} d c^{\prime},
$$

for $\tau>0, \mu \in[-1,1]$ and $c \in[0, \infty)$, and

$$
\begin{aligned}
& Z(0, c, \mu)-(1-\alpha) Z(0, c,-\mu) \\
& \quad-4 \alpha \int_{0}^{\infty} \int_{0}^{1} c^{\prime 3} e^{-c^{\prime 2}} Z\left(0, c^{\prime},-\mu^{\prime}\right) \mu^{\prime} d \mu^{\prime} d c^{\prime} \\
& \quad=R(c, \mu),
\end{aligned}
$$

for $\mu \in(0,1]$ and $c \in[0, \infty)$. Here

$$
R(c, \mu)=(2-\alpha)\left(c^{2}-5 / 2\right) \frac{c \mu}{\eta(c)}+\frac{4 \alpha}{3} \Gamma,
$$

where

$$
\Gamma=\int_{0}^{\infty} \frac{c^{4}}{\eta(c)} e^{-c^{2}}\left(c^{2}-5 / 2\right) d c .
$$


Our basic statement of the problem to be solved is now complete, and so we proceed with our solution; however, before making use of our version of the discrete-ordinates method, we introduce some elementary transformations that will facilitate the development of the final results.

\section{BASIC TRANSFORMATIONS}

Rather than deal explicitly with Eqs. (32) and (33), we choose to follow Busbridge ${ }^{17}$ and to introduce the convenient change of variables

$$
\xi=\frac{c \mu}{\eta(c)}
$$

and

$$
\gamma=\sup \{c / \eta(c)\} .
$$

And so now if we go back to Eq. (32) and introduce the decomposition

$$
\begin{aligned}
Z[\tau, c, \xi \eta(c) / c]= & G_{1}(\tau, \xi)+\xi \eta(c) G_{2}(\tau, \xi) \\
& +\left(c^{2}-\omega\right) G_{3}(\tau, \xi)
\end{aligned}
$$

we find, after an interchange of orders of integration,

$$
\begin{aligned}
\xi \frac{\partial}{\partial \tau} G_{i}(\tau, \xi)+G_{i}(\tau, \xi)= & \int_{-\gamma}^{\gamma}\left[\psi_{i, 1}\left(\xi^{\prime}\right) G_{1}\left(\tau, \xi^{\prime}\right)\right. \\
& +\psi_{i, 2}\left(\xi^{\prime}\right) G_{2}\left(\tau, \xi^{\prime}\right) \\
& \left.+\psi_{i, 3}\left(\xi^{\prime}\right) G_{3}\left(\tau, \xi^{\prime}\right)\right] d \xi^{\prime}
\end{aligned}
$$

for $i=1,2,3$. Here

$$
\begin{aligned}
& \psi_{1,1}(\xi)=\frac{\beta_{0}}{2} \int_{M_{\xi}} c e^{-c^{2}} \eta^{2}(c) d c, \\
& \psi_{1,2}(\xi)=\frac{\beta_{0} \xi}{2} \int_{M_{\xi}} c e^{-c^{2}} \eta^{3}(c) d c, \\
& \psi_{1,3}(\xi)=\frac{\beta_{0}}{2} \int_{M_{\xi}} c e^{-c^{2}} \eta^{2}(c)\left(c^{2}-\omega\right) d c, \\
& \psi_{2,1}(\xi)=\frac{\beta_{1} \xi}{2} \int_{M_{\xi}} c e^{-c^{2}} \eta^{3}(c) d c, \\
& \psi_{2,2}(\xi)=\frac{\beta_{1} \xi^{2}}{2} \int_{M_{\xi}} c e^{-c^{2}} \eta^{4}(c) d c, \\
& \psi_{2,3}(\xi)=\frac{\beta_{1} \xi}{2} \int_{M_{\xi}} c e^{-c^{2}} \eta^{3}(c)\left(c^{2}-\omega\right) d c, \\
& \psi_{3,1}(\xi)=\frac{\beta_{2}}{2} \int_{M_{\xi}} c e^{-c^{2}} \eta^{2}(c)\left(c^{2}-\omega\right) d c, \\
& \psi_{M_{\xi}} c e^{-c^{2}} \eta^{3}(c)\left(c^{2}-\omega\right) d c
\end{aligned}
$$

and

$$
\psi_{3,3}(\xi)=\frac{\beta_{2}}{2} \int_{M_{\xi}} c e^{-c^{2}} \eta^{2}(c)\left(c^{2}-\omega\right)^{2} d c,
$$

where

$$
c \in M_{\xi} \quad \text { if } \frac{\eta(c)|\xi|}{c} \leqslant 1 .
$$

In regard to boundary conditions, we substitute Eq. (37) into Eq. (33) to obtain

$$
\begin{array}{r}
G_{1}(0, \xi)-(1-\alpha) G_{1}(0,-\xi)-\Delta \\
=\frac{4 \alpha}{3} \Gamma+(2-\alpha)(\omega-5 / 2) \xi,
\end{array}
$$

$G_{2}(0, \xi)+(1-\alpha) G_{2}(0,-\xi)=0$,

and

$$
G_{3}(0, \xi)-(1-\alpha) G_{3}(0,-\xi)=(2-\alpha) \xi
$$

for $\xi \in(0, \gamma]$. Here the diffuse term in Eq. (41a) is given by

$$
\begin{aligned}
\Delta= & \frac{8 \alpha}{\beta_{0}} \int_{0}^{\gamma}\left[\psi_{1,1}(\xi) G_{1}(0,-\xi)-\psi_{1,2}(\xi) G_{2}(0,-\xi)\right. \\
& \left.+\psi_{1,3}(\xi) G_{3}(0,-\xi)\right] \xi d \xi .
\end{aligned}
$$

We now introduce the vector-valued function $\mathbf{G}(\tau, \xi)$, with components $G_{i}(\tau, \xi), i=1,2,3$, and write Eq. (38) as

$$
\xi \frac{\partial}{\partial \tau} \mathbf{G}(\tau, \xi)+\mathbf{G}(\tau, \xi)=\int_{-\gamma}^{\gamma} \mathbf{\Psi}\left(\xi^{\prime}\right) \mathbf{G}\left(\tau, \xi^{\prime}\right) d \xi^{\prime},
$$

where the $3 \times 3$ matrix $\boldsymbol{\Psi}(\xi)$ has components $\psi_{i, j}(\xi)$. To have our boundary conditions in vector form, we rewrite Eqs. (41) as

$$
\begin{aligned}
& \mathbf{G}(0, \xi)-(1-\alpha) \mathbf{S G}(0,-\xi) \\
& -2 \alpha \int_{0}^{\gamma} \mathbf{Y}\left(\xi^{\prime}\right) \mathbf{G}\left(0,-\xi^{\prime}\right) \xi^{\prime} d \xi^{\prime}=\mathbf{R}(\xi)
\end{aligned}
$$

for $\xi \in(0, \gamma]$. Here

$$
\begin{aligned}
& \mathbf{S}=\operatorname{diag}\{1,-1,1\}, \\
& \mathbf{Y}(\xi)=\frac{4}{\beta_{0}}\left[\begin{array}{ccc}
\psi_{1,1}(\xi) & -\psi_{1,2}(\xi) & \psi_{1,3}(\xi) \\
0 & 0 & 0 \\
0 & 0 & 0
\end{array}\right]
\end{aligned}
$$

and

$$
\mathbf{R}(\xi)=\left[\begin{array}{c}
(2-\alpha)(\omega-5 / 2) \xi+(4 / 3) \alpha \Gamma \\
0 \\
(2-\alpha) \xi
\end{array}\right] .
$$

And so we seek a bounded (as $\tau \longrightarrow \infty$ ) solution of Eq. (43) that satisfies Eq. (44). Of course, once we have solved the $\mathbf{G}$ problem, we can use Eq. (37) to rewrite Eqs. (30) and (31) as

$$
\begin{aligned}
N_{*}(\tau)= & (K / \sigma)\left\{-\tau+\frac{1}{\pi^{1 / 2}} \int_{-\gamma}^{\gamma}\left[n_{1}(\xi) G_{1}(\tau, \xi)\right.\right. \\
& \left.\left.+n_{2}(\xi) G_{2}(\tau, \xi)+n_{3}(\xi) G_{3}(\tau, \xi)\right] d \xi\right\}
\end{aligned}
$$




$$
\begin{aligned}
T_{*}(\tau)= & (K / \sigma)\left\{\tau+\frac{2}{3 \pi^{1 / 2}} \int_{-\gamma}^{\gamma}\left[t_{1}(\xi) G_{1}(\tau, \xi)\right.\right. \\
& \left.\left.+t_{2}(\xi) G_{2}(\tau, \xi)+t_{3}(\xi) G_{3}(\tau, \xi)\right] d \xi\right\},
\end{aligned}
$$

where

$$
\begin{aligned}
& n_{1}(\xi)=2 \int_{M_{\xi}} c e^{-c^{2}} \eta(c) d c, \\
& n_{2}(\xi)=2 \xi \int_{M_{\xi}} c e^{-c^{2}} \eta^{2}(c) d c,
\end{aligned}
$$

and

$$
n_{3}(\xi)=2 \int_{M_{\xi}} c e^{-c^{2}} \eta(c)\left(c^{2}-\omega\right) d c,
$$

and also where

$$
\begin{aligned}
& t_{1}(\xi)=2 \int_{M_{\xi}} c e^{-c^{2}} \eta(c)\left(c^{2}-3 / 2\right) d c \\
& t_{2}(\xi)=2 \xi \int_{M_{\xi}} c e^{-c^{2}} \eta^{2}(c)\left(c^{2}-3 / 2\right) d c
\end{aligned}
$$

and

$$
t_{3}(\xi)=2 \int_{M_{\xi}} c e^{-c^{2}} \eta(c)\left(c^{2}-\omega\right)\left(c^{2}-3 / 2\right) d c .
$$

It is clear that the scale factor $\sigma$ will have a fundamental effect on our reported numerical results, and since there already exist various possibilities in the literature concerning the definition of an appropriate mean-free path, we elect here to use one of Loyalka's choices ${ }^{9}$ for scaling our results. We therefore define

$$
\sigma=\epsilon_{t}=\frac{16}{15} \pi^{-1 / 2} \int_{0}^{\infty} \eta^{-1}(c) c^{4} e^{-c^{2}}\left(c^{2}-5 / 2\right)^{2} d c
$$

for all models we consider. As noted by Loyalka, ${ }^{9}$ the use of $\sigma=\epsilon_{t}$ corresponds to measuring our spatial variable $x$ in terms of a mean-free path $l_{t}$ that is defined in terms of the thermal conductivity.

\section{THE DISCRETE-ORDINATES SOLUTION}

We note first of all that the characteristic matrix $\boldsymbol{\Psi}(\xi)$, as defined by Eqs. (39), is not symmetric. We note also that $\boldsymbol{\Psi}(\xi) \neq \boldsymbol{\Psi}(-\xi)$, and so we write our discrete-ordinates version of Eq. (43) as

$$
\begin{aligned}
& \pm \xi_{i} \frac{d}{d \tau} \mathbf{G}\left(\tau, \pm \xi_{i}\right)+\mathbf{G}\left(\tau, \pm \xi_{i}\right) \\
& \quad=\sum_{k=1}^{N} w_{k}\left[\mathbf{\Psi}\left(\xi_{k}\right) \mathbf{G}\left(\tau, \xi_{k}\right)+\mathbf{\Psi}\left(-\xi_{k}\right) \mathbf{G}\left(\tau,-\xi_{k}\right)\right]
\end{aligned}
$$

for $i=1,2, \ldots, N$. In writing Eqs. (53) as we have, we clearly are considering that the $N$ quadrature points $\left\{\xi_{k}\right\}$ and the $N$ weights $\left\{w_{k}\right\}$ are defined for use on the integration interval $[0, \gamma]$. We note that it is to this feature of using a "half- range" quadrature scheme that we partially attribute the especially good accuracy we have obtained from the solution reported here. Continuing, we substitute

$$
\mathbf{G}\left(\tau, \pm \xi_{i}\right)=\boldsymbol{\Phi}\left(\nu, \pm \xi_{i}\right) e^{-\tau / \nu}
$$

into Eqs. (53) to find

$$
\begin{aligned}
\left(\nu \mp \xi_{i}\right) \boldsymbol{\Phi}\left(\nu, \pm \xi_{i}\right)= & \nu \sum_{k=1}^{N} w_{k}\left[\boldsymbol{\Psi}\left(\xi_{k}\right) \boldsymbol{\Phi}\left(\nu, \xi_{k}\right)\right. \\
& \left.+\boldsymbol{\Psi}\left(-\xi_{k}\right) \boldsymbol{\Phi}\left(\nu,-\xi_{k}\right)\right]
\end{aligned}
$$

for $i=1,2, \ldots, N$. Now let

$\boldsymbol{\Phi}_{+}(\nu)=\left[\begin{array}{llll}\boldsymbol{\Phi}^{T}\left(\nu, \xi_{1}\right) & \boldsymbol{\Phi}^{T}\left(\nu, \xi_{2}\right) & \cdots & \boldsymbol{\Phi}^{T}\left(\nu, \xi_{N}\right)\end{array}\right]^{T}$,

$\boldsymbol{\Phi}_{-}(\nu)=\left[\begin{array}{llll}\boldsymbol{\Phi}^{T}\left(\nu,-\xi_{1}\right) & \boldsymbol{\Phi}^{T}\left(\nu,-\xi_{2}\right) & \cdots & \boldsymbol{\Phi}^{T}\left(\nu,-\xi_{N}\right)\end{array}\right]^{T}$,

and

$$
\mathbf{M}=\operatorname{diag}\left\{\xi_{1} \mathbf{I}, \xi_{2} \mathbf{I}, \ldots, \xi_{N} \mathbf{I}\right\},
$$

where $\mathbf{I}$ is the $3 \times 3$ identity matrix. In addition we let $\mathbf{W}_{+}$ and $\mathbf{W}_{\text {- denote }} 3 N \times 3 N$ matrices each $3 \times 3 N$ row of which is, respectively,

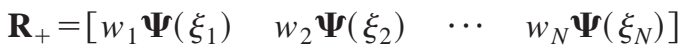

and

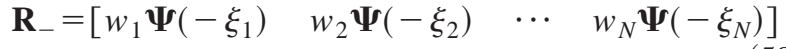

so that we can write Eqs. (55) as

$$
\nu \boldsymbol{\Phi}_{+}(\nu)-\mathbf{M} \boldsymbol{\Phi}_{+}(\nu)=\nu\left[\mathbf{W}_{+} \boldsymbol{\Phi}_{+}(\nu)+\mathbf{W}_{-} \boldsymbol{\Phi}_{-}(\nu)\right]
$$

and

$$
\nu \boldsymbol{\Phi}_{-}(\nu)+\mathbf{M} \boldsymbol{\Phi}_{-}(\nu)=\nu\left[\mathbf{W}_{+} \boldsymbol{\Phi}_{+}(\nu)+\mathbf{W}_{-} \boldsymbol{\Phi}_{-}(\nu)\right] .
$$

At this point we find, after noting some basic properties of $\boldsymbol{\Psi}(\xi)$, that we can write

$$
\mathbf{W}_{-}=\mathbf{D} \mathbf{W}_{+} \mathbf{D}
$$

where the $3 N \times 3 N$ diagonal matrix $\mathbf{D}$ can be written as

$$
\mathbf{D}=\operatorname{diag}\{\mathbf{S}, \mathbf{S}, \ldots, \mathbf{S}\}
$$

with $\mathbf{S}$ as given by Eq. (45). We now multiply Eq. (60b) by $\mathbf{D}$ and rewrite Eqs. (60) as

$$
\nu \boldsymbol{\Phi}_{+}(\nu)-\mathbf{M} \boldsymbol{\Phi}_{+}(\nu)=\nu\left[\mathbf{W}_{+} \boldsymbol{\Phi}_{+}(\nu)+\mathbf{D} \mathbf{W}_{+} \mathbf{D} \boldsymbol{\Phi}_{-}(\nu)\right]
$$

and

$\nu \mathbf{D} \Phi_{-}(\nu)+\mathbf{M D} \Phi_{-}(\nu)=\nu\left[\mathbf{D} \mathbf{W}_{+} \boldsymbol{\Phi}_{+}(\nu)+\mathbf{W}_{+} \mathbf{D} \Phi_{-}(\nu)\right]$.

Now let

$$
\mathbf{U}=\boldsymbol{\Phi}_{+}(\nu)+\mathbf{D} \Phi_{-}(\nu)
$$

and

$$
\mathbf{V}=\boldsymbol{\Phi}_{+}(\nu)-\mathbf{D} \boldsymbol{\Phi}_{-}(\nu)
$$


and add Eqs. (63) to obtain

$$
\nu\left[\mathbf{I}-(\mathbf{I}+\mathbf{D}) \mathbf{W}_{+}\right] \mathbf{U}=\mathbf{M V},
$$

where now I is the $3 N \times 3 N$ identity matrix. We also compute the difference between Eqs. (63) to find

$$
\nu\left[\mathbf{I}-(\mathbf{I}-\mathbf{D}) \mathbf{W}_{+}\right] \mathbf{V}=\mathbf{M U} .
$$

We now can eliminate $\mathbf{V}(\nu)$ between Eqs. (65) and (66) to find the eigenvalue problem

$$
\mathbf{A U}=\lambda \mathbf{U},
$$

where $\lambda=1 / \nu^{2}$ and

$$
\mathbf{A}=\mathbf{M}^{-1}\left[\mathbf{I}-(\mathbf{I}-\mathbf{D}) \mathbf{W}_{+}\right] \mathbf{M}^{-1}\left[\mathbf{I}-(\mathbf{I}+\mathbf{D}) \mathbf{W}_{+}\right] .
$$

And so our first computational job is to find the $3 N$ eigenvalues of $\mathbf{A}$. However we first wish to address the issue of infinite values of the separation constant $\nu$ (or equivalently, the eigenvalues of $\mathbf{A}$ that approach zero as $N$ tends to infinity). We first introduce

$$
\boldsymbol{\Lambda}(z)=\mathbf{I}+z \int_{-\gamma}^{\gamma} \boldsymbol{\Psi}(\xi) \frac{d \xi}{\xi-z}
$$

and note that we consider $\Lambda(z)$ to be the exact version of the discrete-ordinates quantity

$$
\boldsymbol{\Omega}(z)=\mathbf{I}+z \sum_{k=1}^{N} w_{k}\left[\boldsymbol{\Psi}\left(\xi_{k}\right) \frac{1}{\xi_{k}-z}-\boldsymbol{\Psi}\left(-\xi_{k}\right) \frac{1}{\xi_{k}+z}\right] .
$$

Since we know that the separation constants $\nu_{j}$ defined by the zeros of $\operatorname{det} \boldsymbol{\Omega}(z)$ are the same as those we compute from the eigenvalues of the matrix $\mathbf{A}$, we base our discussion about the eigenvalues of $\mathbf{A}$ (that accumulate at zero as $N$ tends to infinity) on the zeros of $\operatorname{det} \Lambda(z)$ as $z$ tends to infinity. We find that

$$
\operatorname{det} \Lambda(z) \sim \frac{M}{z^{4}}, \quad M \neq 0,
$$

as $z \rightarrow \infty$, and so we conclude that, as $N$ tends to infinity, $\mathbf{A}$ should have $\lambda=0$ as a (two-fold) repeated eigenvalue. And so instead of using Eq. (54) for the two smallest eigenvalues of $\mathbf{A}$ we use instead the following four exact solutions of Eq. (43):

$$
\mathbf{G}_{1}=\left[\begin{array}{l}
0 \\
0 \\
1
\end{array}\right]
$$

and

$$
\mathbf{G}_{2}=\left[\begin{array}{l}
0 \\
1 \\
0
\end{array}\right]
$$

along with

$$
\mathbf{G}_{3}=\left[\begin{array}{l}
1 \\
0 \\
0
\end{array}\right]
$$

$$
\mathbf{G}_{4}(\tau, \xi)=(\tau-\xi)\left[\begin{array}{c}
\omega-5 / 2 \\
0 \\
1
\end{array}\right]
$$

If we now let

$\mathbf{G}_{ \pm}(\tau)=\left[\begin{array}{llll}\mathbf{G}^{T}\left(\tau, \pm \xi_{1}\right) & \mathbf{G}^{T}\left(\tau, \pm \xi_{2}\right) & \cdots & \mathbf{G}^{T}\left(\tau, \pm \xi_{N}\right)\end{array}\right]^{T}$,

then our discrete-ordinates solution can be written (after we exclude all solutions that are not bounded as $\tau$ tends to infinity) as

$$
\mathbf{G}_{ \pm}(\tau)=A_{1} \boldsymbol{\Phi}_{1}+A_{2} \boldsymbol{\Phi}_{2}+B_{1} \boldsymbol{\Phi}_{3}+\sum_{j=3}^{3 N} A_{j} \boldsymbol{\Phi}_{ \pm}\left(\nu_{j}\right) e^{-\tau / \nu_{j}},
$$

where $B_{1}$ and $A_{j}$, for $j=1,2, \ldots, 3 N$, are arbitrary constants. In addition

$$
\boldsymbol{\Phi}_{j}=\left[\begin{array}{llll}
\mathbf{G}_{j}^{T} & \mathbf{G}_{j}^{T} & \cdots & \mathbf{G}_{j}^{T}
\end{array}\right]^{T}, \quad j=1,2,3,
$$

and the $\boldsymbol{\Phi}_{ \pm}\left(\nu_{j}\right)$ are available from Eqs. (64), (65), and (66). We find

$$
\boldsymbol{\Phi}_{+}\left(\nu_{j}\right)=\frac{1}{2}\left\{\mathbf{I}+\nu_{j} \mathbf{M}^{-1}\left[\mathbf{I}-(\mathbf{I}+\mathbf{D}) \mathbf{W}_{+}\right]\right\} \mathbf{U}_{j}
$$

and

$$
\boldsymbol{\Phi}_{-}\left(\nu_{j}\right)=\frac{1}{2} \mathbf{D}\left\{\mathbf{I}-\nu_{j} \mathbf{M}^{-1}\left[\mathbf{I}-(\mathbf{I}+\mathbf{D}) \mathbf{W}_{+}\right]\right\} \mathbf{U}_{j},
$$

where $\mathbf{U}_{j}$ is the eigenvector of $\mathbf{A}$ that corresponds to the eigenvalue $\lambda_{j}$. Looking back now to Eq. (44), we find, for this formulation, that the boundary condition can be written as

$$
\mathbf{G}_{+}(0)-(1-\alpha) \mathbf{R}_{s} \mathbf{G}_{-}(0)-2 \alpha \mathbf{R}_{d} \mathbf{G}_{-}(0)=\mathbf{R},
$$

where the known right-hand side is given by

$$
\mathbf{R}=\left[\begin{array}{llll}
\mathbf{R}^{T}\left(\xi_{1}\right) & \mathbf{R}^{T}\left(\xi_{2}\right) & \cdots & \mathbf{R}^{T}\left(\xi_{N}\right)
\end{array}\right]^{T} .
$$

Note that $\mathbf{R}(\xi)$ is given by Eq. (47). In addition, we find we can write the specular matrix as

$$
\mathbf{R}_{s}=\operatorname{diag}\{\mathbf{S}, \mathbf{S}, \ldots, \mathbf{S}\},
$$

where $\mathbf{S}$ is given by Eq. (45). Finally, to account for diffuse reflection, $\mathbf{R}_{d}$ is a $3 N \times 3 N$ matrix each $3 \times 3 N$ row of which is given by

$$
\mathbf{R}_{r}=\left[\begin{array}{llll}
w_{1} \xi_{1} \mathbf{Y}\left(\xi_{1}\right) & \omega_{2} \xi_{2} \mathbf{Y}\left(\xi_{2}\right) & \cdots & \omega_{N} \xi_{N} \mathbf{Y}\left(\xi_{N}\right)
\end{array}\right]
$$

where $\mathbf{Y}(\xi)$ is given by Eq. (46). It is clear that Eq. (77) is a general result, but when the exact terms $\boldsymbol{\Phi}_{1}, \boldsymbol{\Phi}_{2}$, and $\boldsymbol{\Phi}_{3}$ are used in Eq. (44) the integrals resulting from the diffuse reflection can be done exactly. Finally, we note that since $\boldsymbol{\Phi}_{3}$ satisfies the homogeneous version of Eq. (77) the constant $B_{1}$ cannot be determined from that equation. However, we can impose on our solution the additional (arbitrary) normalization condition

$$
\lim _{\tau \rightarrow \infty}\left[N_{*}(\tau)+T_{*}(\tau)\right]=\Gamma_{*} .
$$

At this point, we follow other works ${ }^{15,18,19}$ and use $\Gamma_{*}=0$ which, when we consider Eqs. (48), (49), and (73), yields

$$
B_{1}=(\omega-5 / 2) A_{1} \text {. }
$$


Considering now the quantities we wish to evaluate, we substitute Eq. (73) into Eqs. (48) and (49) to find, after using Eq. (82),

$$
\begin{aligned}
N_{*}(\tau)= & (K / \sigma)\left\{-\tau-A_{1}+\frac{1}{\pi^{1 / 2}} \sum_{j=3}^{3 N} A_{j}\left[\mathbf{N}_{+} \boldsymbol{\Phi}_{+}\left(\nu_{j}\right)\right.\right. \\
& \left.\left.+\mathbf{N}_{-} \boldsymbol{\Phi}_{-}\left(\nu_{j}\right)\right] e^{-\tau / \nu_{j}}\right\}
\end{aligned}
$$

and

$$
\begin{aligned}
T_{*}(\tau)= & (K / \sigma)\left\{\tau+A_{1}+\frac{2}{3 \pi^{1 / 2}} \sum_{j=3}^{3 N} A_{j}\left[\mathbf{T}_{+} \mathbf{\Phi}_{+}\left(\nu_{j}\right)\right.\right. \\
& \left.\left.+\mathbf{T}_{-} \boldsymbol{\Phi}_{-}\left(\nu_{j}\right)\right] e^{-\tau / \nu_{j}}\right\},
\end{aligned}
$$

where

$$
\mathbf{N}_{ \pm}=\left[\begin{array}{llll}
w_{1} \mathbf{N}\left( \pm \xi_{1}\right) & w_{2} \mathbf{N}\left( \pm \xi_{2}\right) & \cdots & w_{N} \mathbf{N}\left( \pm \xi_{N}\right)
\end{array}\right]
$$

and

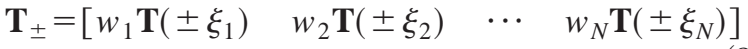

with

$$
\mathbf{N}(\xi)=\left[n_{1}(\xi) \quad n_{2}(\xi) \quad n_{3}(\xi)\right]
$$

and

$$
\mathbf{T}(\xi)=\left[t_{1}(\xi) \quad t_{2}(\xi) \quad t_{3}(\xi)\right]
$$

Note that the components of the vectors introduced in Eqs. (86) are defined by Eqs. (50) and (51). We note also that in obtaining Eqs. (83) and (84) from Eqs. (48) and (49), we have analytically integrated the first three terms of Eq. (73), but we have used our defined quadrature scheme to integrate the remaining terms. Now putting Eqs. (83) and (84) back in terms of the $x$ variable, we find

$$
\begin{aligned}
N(x)= & -x-A_{1} / \sigma+\frac{1}{\sigma \pi^{1 / 2}} \sum_{j=3}^{3 N} A_{j}\left[\mathbf{N}_{+} \boldsymbol{\Phi}_{+}\left(\nu_{j}\right)\right. \\
& \left.+\mathbf{N}_{-} \boldsymbol{\Phi}_{-}\left(\nu_{j}\right)\right] e^{-\sigma x / \nu_{j}}
\end{aligned}
$$

and

$$
\begin{aligned}
T(x)= & x+A_{1} / \sigma+\frac{2}{3 \sigma \pi^{1 / 2}} \sum_{j=3}^{3 N} A_{j}\left[\mathbf{T}_{+} \mathbf{\Phi}_{+}\left(\nu_{j}\right)\right. \\
& \left.+\mathbf{T}_{-} \mathbf{\Phi}_{-}\left(\nu_{j}\right)\right] e^{-\sigma x / \nu_{j}},
\end{aligned}
$$

where we have imposed the normalization $K=1$. If now we let

$$
T_{\text {asy }}(x)=x+A_{1} / \sigma
$$

and define the temperature-jump coefficient $\zeta$ by

$$
T_{\text {asy }}(0)=\left.\zeta \frac{d}{d x} T_{\text {asy }}(x)\right|_{x=0},
$$

then clearly

$$
\zeta=A_{1} / \sigma
$$

To be very clear, we note that the constant $\Gamma_{*}$ introduced in Eq. (81) does not affect the temperature-jump coefficient or the temperature perturbation $T(x)$. In fact, another choice of $\Gamma_{*}$ would change only the density perturbations $N(x)$ by the addition of a constant factor.

\section{SPECIAL CASES}

Having developed our general solution to the temperature-jump problem for the CLF model of the linearized Boltzmann equation, we are ready to list the specific forms of certain basic quantities for the three special cases we consider in this work.

\section{A. Constant collision frequency}

For this case, the classical BGK model, we write

$$
\eta(c)=1,
$$

and so we find

$$
\begin{aligned}
& \gamma=\infty, \\
& \Gamma=0, \\
& \omega=3 / 2, \\
& \beta_{0}=4 / \pi^{1 / 2},
\end{aligned}
$$

and

$$
\sigma=1
$$




$$
\boldsymbol{\Psi}(\xi)=\frac{e^{-\xi^{2}}}{\pi^{1 / 2}}\left[\begin{array}{ccc}
1 & \xi & \xi^{2}-1 / 2 \\
2 \xi & 2 \xi^{2} & 2 \xi\left(\xi^{2}-1 / 2\right) \\
(2 / 3)\left(\xi^{2}-1 / 2\right) & (2 / 3) \xi\left(\xi^{2}-1 / 2\right) & (2 / 3)\left(\xi^{4}-\xi^{2}+5 / 4\right)
\end{array}\right]
$$

We can also use Eqs. (50) and (51) to write Eqs. (86), for this case, as

$$
\mathbf{N}(\xi)=e^{-\xi^{2}}\left[\begin{array}{lll}
1 & \xi & \xi^{2}-1 / 2
\end{array}\right]
$$

and

$$
\mathbf{T}(\xi)=e^{-\xi^{2}}\left[\xi^{2}-1 / 2 \quad \xi\left(\xi^{2}-1 / 2\right) \quad \xi^{4}-\xi^{2}+5 / 4\right] .
$$

\section{B. The Williams model}

For this case we write

$$
\eta(c)=c,
$$

and so we find

$$
\begin{aligned}
& \gamma=1, \\
& \Gamma=-1 / 4, \\
& \omega=2, \\
& \beta_{0}=2,
\end{aligned}
$$

and

$$
\sigma=(6 / 5) \pi^{-1 / 2} .
$$

In addition, we find from Eqs. (39)

$$
\boldsymbol{\Psi}(\xi)=\frac{1}{2}\left[\begin{array}{ccc}
1 & (3 / 4) \pi^{1 / 2} \xi & 0 \\
(9 / 8) \pi^{1 / 2} \xi & 3 \xi^{2} & (9 / 16) \pi^{1 / 2} \xi \\
0 & (3 / 16) \pi^{1 / 2} \xi & 1
\end{array}\right]
$$

Again we can use Eqs. (50) and (51) to write Eqs. (86), for this case, as

$$
\mathbf{N}(\xi)=\left[(1 / 2) \pi^{1 / 2} \quad \xi \quad-(1 / 4) \pi^{1 / 2}\right]
$$

and

$$
\mathbf{T}(\xi)=\left[\begin{array}{lll}
0 & \xi / 2 & (3 / 4) \pi^{1 / 2}
\end{array}\right] .
$$

\section{The rigid-sphere model}

For the rigid-sphere model, we follow Loyalka and Hickey ${ }^{11}$ and write

$$
\eta(c)=\left(2 c+\frac{1}{c}\right) \frac{\pi^{1 / 2}}{2} \operatorname{erf}(c)+e^{-c^{2}},
$$

where $\operatorname{erf}(c)$ is the error function. Here we find the exact results

$$
\gamma=1 / \pi^{1 / 2}
$$

and

$$
\omega=7 / 4,
$$

and we have used the software package MAPLE $\mathrm{V}$ to find the numerical values

$$
\begin{aligned}
& \beta_{0}=0.7978845608029, \\
& \Gamma=-0.06063367084623,
\end{aligned}
$$

and

$$
\sigma=0.2753345876233 .
$$

In regard to Eqs. (39), (50), and (51), we have used numerical methods to evaluate the various functions required to establish $\boldsymbol{\Psi}(\xi), \mathbf{N}(\xi)$, and $\mathbf{T}(\xi)$. As discussed in a previous work $^{12}$ concerning Kramers' problem, we let

$$
f(c)=\frac{c}{\eta(c)}
$$

and note that we can show, for the considered case, that $f^{\prime}(c)>0$, for $c \geqslant 0$ and so the inverse function

$$
m(\xi)=f^{-1}(|\xi|), \quad \xi \in[-\gamma, \gamma],
$$

exists, and thus we can write the required functions (written symbolically as)

$$
P(\xi)=\int_{M_{\xi}} p(c) d c
$$

as

$$
P(\xi)=\int_{m(\xi)}^{\infty} p(c) d c,
$$

which can be evaluated numerically once $m(\xi)$ is available; as before ${ }^{12}$ we use Newton's method to establish the required numerical values of $m(\xi)$.

\section{NUMERICAL RESULTS}

The first thing we must do is to define the quadrature scheme to be used in our discrete-ordinates solution, and, since we have considered three different cases, to which we refer as case 1 , case 2 , and case 3 while meaning, respectively, the BGK model, the Williams model, and the rigidsphere model, we have used three different maps. For case 1, we used the (nonlinear) transformation

$$
u(\xi)=\exp \{-\xi\}
$$

to map $\xi \in[0, \infty)$ into $u \in[0,1]$, and we then used the GaussLegendre scheme mapped (linearly) onto the interval $[0,1]$. For cases 2 and 3 we simply mapped the Gauss-Legendre scheme onto, respectively, the intervals $[0,1]$ and $\left[0, \pi^{-1 / 2}\right]$. Having defined our quadrature scheme, we used the driver program RG from the EISPACK collection ${ }^{20}$ to find the eigenvalues and eigenvectors defined by Eq. (67). And so, after using the subroutines DGECO and DGESL from the LINPACK package $^{21}$ to solve the linear system derived from Eq. (77) to 
TABLE I. The temperature-jump coefficient $\zeta$.

\begin{tabular}{lcccccc}
\hline \hline Model & $\alpha=0.1$ & $\alpha=0.3$ & $\alpha=0.5$ & $\alpha=0.6$ & $\alpha=0.7$ & $\alpha=0.9$ \\
\hline Case 1 & 21.45012 & 6.630514 & 3.629125 & 2.867615 & 2.317534 & 1.570264 \\
Case 2 & 21.19359 & 6.406417 & 3.435960 & 2.689383 & 2.153897 & 1.434848 \\
Case 3 & 21.24657 & 6.452894 & 3.476180 & 2.726563 & 2.188095 & 1.463247 \\
\hline \hline
\end{tabular}

find the constants $A_{j}$, for $j=1,2, \ldots, 3 N$, we consider our solution complete. Finally, but importantly, we have found, that some elements of the matrix-valued function $\boldsymbol{\Psi}(\xi)$ as defined by Eqs. (39) can be essentially zero (from a computational point-of-view). In such cases, we found that by defining an element to be precisely zero when that element is less than, say, $\epsilon=10^{-50}$, we greatly increased the ability of the linearalgebra package to yield the required number of independent eigenvectors when there is a (nearly) repeated eigenvalue.

To complete our work we list in Tables I and II some results obtained from our FORTRAN implementation of the developed solution of the temperature-jump problem for the three explicitly considered cases. We note that our results are given with what we believe to be seven, in Table I, and six, in Table II, figures of accuracy. While we have no proof of the accuracy achieved in this work, we have done some things to support the confidence we have. First of all our results for case 1 agree perfectly with some (quasi) independent calculations ${ }^{15}$ done previously. In addition we found agreement, to three figures, with the value of $\zeta$ for case 2 , with $\alpha=1.0$, that was reported by Cassell and Williams. ${ }^{22} \mathrm{We}$ also found apparent convergence in our numerical results as we increased $N$, the number of quadrature points used, and to reduce the possibility of FORTRAN errors, we have implemented two independent versions of the algorithm. Finally, we note that for case 2, the Williams model, the three-vector $\mathbf{G}$ problem can, as discussed by Williams and Cassell, ${ }^{22}$ be solved as three consecutive scalar problems. This approach has been used by Bartz ${ }^{23}$ to confirm all of the results given in Tables I and II that refer to case 2 .

We have typically used $N=50$ to generate results for the temperature-jump coefficient $\zeta$ and the temperature and density perturbations good to, say, five or six significant figures, and so we note that our FORTRAN implementation (no special effort was made to make the code especially efficient) of our discrete-ordinates solution (with $N=50$ ) runs in a few seconds on a $400 \mathrm{MHz}$ Pentium-based PC.

To have some idea of the merits of the CLF model, as we have used it here, we note that Loyalka ${ }^{9}$ and Sone, Ohwada, and Aoki $^{10}$ give, respectively, for the case $\alpha=1$, the results $\zeta=1.2486$ and $\zeta=1.2482$ for the case of the linearized Boltzmann equation relevant to hard-sphere collisions. If we consider these results to be the best available for the problem as defined in this work, then our use of the CLF model for the rigid-sphere case $(\zeta=1.206526)$ provides a modest improvement (in regard to the temperature-jump coefficient for the case $\alpha=1)$ over the classical BGK model ( $\zeta=1.302716)$.

\section{FINAL COMMENTS}

In concluding this work, we note that we have been able to extend the use of our analytical version of the discreteordinates method to solve the temperature-jump problem for a general version of the variable collision frequency model.

TABLE II. The temperature and density perturbations for the case $\alpha=0.5$.

\begin{tabular}{|c|c|c|c|c|c|c|}
\hline \multirow[b]{2}{*}{$x$} & \multicolumn{2}{|c|}{ Case 1} & \multicolumn{2}{|c|}{ Case 2} & \multicolumn{2}{|c|}{ Case 3} \\
\hline & $T(x)$ & $N(x)$ & $T(x)$ & $N(x)$ & $T(x)$ & $N(x)$ \\
\hline 0.0 & 2.91597 & -3.07437 & 3.10167 & -3.27399 & 3.00508 & -3.16704 \\
\hline 0.1 & 3.18042 & -3.31664 & 3.30560 & -3.42542 & 3.23434 & -3.35822 \\
\hline 0.2 & 3.36278 & -3.48323 & 3.45447 & -3.54940 & 3.39748 & -3.50093 \\
\hline 0.3 & 3.52167 & -3.62947 & 3.58758 & -3.66556 & 3.54146 & -3.63010 \\
\hline 0.4 & 3.66754 & -3.76478 & 3.71209 & -3.77746 & 3.67483 & -3.75193 \\
\hline 0.5 & 3.80489 & -3.89310 & 3.83110 & -3.88665 & 3.80132 & -3.86906 \\
\hline 0.6 & 3.93615 & -4.01653 & 3.94628 & -3.99397 & 3.92295 & -3.98291 \\
\hline 0.7 & 4.06283 & -4.13633 & 4.05866 & -4.09990 & 4.04097 & -4.09435 \\
\hline 0.8 & 4.18593 & -4.25334 & 4.16891 & -4.20480 & 4.15620 & -4.20394 \\
\hline 0.9 & 4.30614 & -4.36814 & 4.27749 & -4.30888 & 4.26921 & -4.31208 \\
\hline 1.0 & 4.42400 & -4.48113 & 4.38475 & -4.41233 & 4.38044 & -4.41906 \\
\hline 2.0 & 5.52928 & -5.55674 & 5.42014 & -5.42888 & 5.44006 & -5.45530 \\
\hline 3.0 & 6.57466 & -6.58912 & 6.43030 & -6.43349 & 6.46062 & -6.46735 \\
\hline 4.0 & 7.59758 & -7.60560 & 7.43378 & -7.43503 & 7.46901 & -7.47216 \\
\hline 5.0 & 8.61013 & -8.61476 & 8.43508 & -8.43559 & 8.47273 & -8.47426 \\
\hline 6.0 & 9.61737 & -9.62011 & 9.43559 & -9.43581 & 9.47447 & -9.47523 \\
\hline 7.0 & 10.6217 & -10.6234 & 10.4358 & -10.4359 & 10.4753 & -10.4757 \\
\hline 8.0 & 11.6243 & -11.6254 & 11.4359 & -11.4359 & 11.4757 & -11.4759 \\
\hline 9.0 & 12.6260 & -12.6267 & 12.4359 & -12.4359 & 12.4759 & -12.4761 \\
\hline 10.0 & 13.6271 & -13.6275 & 13.4359 & -13.4360 & 13.4761 & -13.4761 \\
\hline 20.0 & 23.6291 & -23.6291 & 23.4360 & -23.4360 & 23.4762 & -23.4762 \\
\hline
\end{tabular}


In addition to a formulation valid for a general form of the collision frequency, the algorithm was implemented to yield high-quality numerical results for three well-regarded forms. Since the reported solution is easy to evaluate and yields excellent numerical results, and since the developed code runs typically in a few seconds on a $400 \mathrm{MHz}$ Pentium-based PC, we consider the solution ready for additional applications. It is clear that the formalism reported here can readily be used to solve other classical problems, in semi-infinite media and for plane-parallel channel flow, in rarefied-gas dynamics, when one of the three explicitly developed variants of the CLF model equation is required to give (perhaps) more realistic results than the standard BGK model can provide. And finally, since the development reported here is also general, we believe that our solution can immediately be used with forms of the collision frequency additional to the three special cases considered.

\section{ACKNOWLEDGMENTS}

The authors take this opportunity to thank S. K. Loyalka, F. Sharipov, and M. M. R. Williams for some helpful discussions concerning this (and other) work. In addition, it is noted that the work of L.B.B. was supported in part by CNPq of Brazil.

${ }^{1}$ C. Cercignani, Mathematical Methods in Kinetic Theory (Plenum, New York, 1969).

${ }^{2}$ C. Cercignani, The Boltzmann Equation and its Applications (SpringerVerlag, New York, 1988).

${ }^{3}$ M. M. R. Williams, Mathematical Methods in Particle Transport Theory (Butterworth, London, 1971).

${ }^{4}$ F. Sharipov and V. Seleznev, "Data on internal rarefied gas flows," J. Phys. Chem. Ref. Data 27, 657 (1998).

${ }^{5}$ P. L. Bhatnagar, E. P. Gross, and M. Krook, "A model for collision processes in gases. I. Small amplitude processes in charged and neutral onecomponent systems," Phys. Rev. 94, 511 (1954).

${ }^{6} \mathrm{M}$. M. R. Williams, "Boundary-value problems in the kinetic theory of gases Part I. Slip flow," J. Fluid Mech. 36, 145 (1969).
${ }^{7}$ C. Cercignani, "The method of elementary solutions for kinetic models with velocity-dependent collision frequency," Ann. Phys. (N.Y.) 40, 469 (1966).

${ }^{8}$ S. K. Loyalka and J. H. Ferziger, "Model dependence of the temperature slip coefficient," Phys. Fluids 11, 1668 (1968).

${ }^{9}$ S. K. Loyalka, "Temperature jump and thermal creep slip: Rigid sphere gas," Phys. Fluids A 1, 403 (1989).

${ }^{10}$ Y. Sone, T. Ohwada, and K. Aoki, "Temperature jump and Knudsen layer in rarefied gas over a plane wall: Numerical analysis of the linearized Boltzmann equation for hard-sphere molecules," Phys. Fluids A 1, 363 (1989).

${ }^{11}$ S. K. Loyalka and K. A. Hickey, "The Kramers problem: Velocity slip and defect for a hard sphere gas with arbitrary accommodation," Z. Angew. Math. Phys. 41, 245 (1990).

${ }^{12}$ C. E. Siewert, "Kramers' problem for a variable collision frequency model," Eur. J. Appl. Math. 12, 179 (2001).

${ }^{13}$ L. B. Barichello and C. E. Siewert, "A discrete-ordinates solution for a non-grey model with complete frequency redistribution," J. Quant. Spectrosc. Radiat. Transf. 62, 665 (1999).

${ }^{14}$ S. Chandrasekhar, Radiative Transfer (Oxford University Press, London, 1950).

${ }^{15}$ L. B. Barichello and C. E. Siewert, "The temperature-jump problem in rarefied-gas dynamics," Eur. J. Appl. Math. 11, 353 (2000).

${ }^{16} \mathrm{P}$. Welander, "On the temperature jump in a rarefied gas," Ark. Fys. 7, 507 (1954).

${ }^{17} \mathrm{I}$. W. Busbridge, "Coherent and non-coherent scattering in the theory of line formation,” Mon. Not. R. Astron. Soc. 113, 52 (1953).

${ }^{18}$ J. T. Kriese, T. S. Chang, and C. E. Siewert, "Elementary solutions of coupled model equations in the kinetic theory of gases," Int. J. Eng. Sci. 12, 441 (1974).

${ }^{19}$ Y. Onishi, "Kinetic theory analysis for temperature and density fields of a slightly rarefied binary gas mixture over a solid wall," Phys. Fluids 9, 226 (1997).

${ }^{20}$ B. T. Smith, J. M. Boyle, J. J. Dongarra, B. S. Garbow, Y. Ikebe, V. C. Klema, and C. B. Moler, Matrix Eigensystem Routines-EISPACK Guide (Springer-Verlag, Berlin, 1976).

${ }^{21}$ J. J. Dongarra, J. R. Bunch, C. B. Moler, and G. W. Stewart, LINPACK User's Guide (SIAM, Philadelphia, 1979).

${ }^{22}$ J. S. Cassell and M. M. R. Williams, "An exact solution of the temperature slip problem in rarefied gases," Transp. Theory Stat. Phys. 2, 61 (1972).

${ }^{23}$ A. C. R. Bartz, M.S. thesis, Programa de Pós-Graduação em Matemática Aplicada, Universidade Federal do Rio Grande do Sul, Brazil, 2000 (in Portuguese). 